\title{
Surface chirality influences molecular rotation upon desorption
}

\author{
Sabine C. Matysik, David J. Wales, and Stephen J. Jenkins ${ }^{a)}$ \\ Department of Chemistry \\ University of Cambridge \\ Lensfield Rd, Cambridge CB2 1EW, UK
}

(Dated: 3 March 2021)

Chiral surfaces offer great potential as a medium for enantioselective synthesis or separation, yet their dynamic enantiospecific interactions with adsorbates are not well understood. Here, the influence of chiral surfaces on the molecular rotations of desorbing molecules is investigated. Formic acid desorption from $\mathrm{Cu}\{531\}$ and $\mathrm{Cu}\{110\}$ serve as model systems for desorption processes of an achiral adsorbate from a chiral and an achiral surface. Our first-principles molecular dynamics study reveals a much larger and more directed angular momentum for molecules desorbing from the chiral surface, and a clear preference for one sense of rotation. This result provides new insight into desorbtion and adsorption processes and propensities on chiral surfaces.

Chirality can manifest itself in both structure and dynamics. While structural chirality is of great interest in many different fields, such as pharmaceutical design, primarily at molecular length scales, chiral motion is harder to analyse, and is often only accounted for as a macroscopic property. Despite this difference in length scales, there have been various attempts to establish causal relationships between them. One such attempt was published recently, reporting targeted formation of chiral supramolecular structures from achiral monomers by applying a vortex motion throughout the aggregation process. ${ }^{1}$ If macroscale rotational chirality can be linked to chiral nanoscale structures, it is likely that such connections can also be found within a single length scale. Stateof-the-art molecular beam experiments have investigated the influence of stereodynamics on surface reactions, and several techniques to select molecules according to their rotational state have been developed. ${ }^{2}$ It has been shown that adsorption and reaction probabilities can differ, depending on the rotational state of the molecule, but up to this point without regard to the sense of the rotation, i.e. its chirality. ${ }^{3}$ Herein, we report such a link between a structurally chiral state of an achiral, surface-adsorbed molecule and its chiral molecular rotation upon desorption. We analyse the desorption of formic acid as a simple achiral molecule from the chiral $\mathrm{R}(\mathrm{D})-\mathrm{Cu}\{531\}$ surface as a model system by means of first-principles molecular dynamics (MD) based on density functional theory (DFT). Chiral surface systems have been studied for some time ${ }^{4}$ but coupling of surface asymmetry to the rotational motion of adsorbates has not, to the best of our knowledge, been previously discussed.

Extracting reactive trajectories that are connected to an adsorption and dissociation event from a sample with random initial parameters is computationally very expensive at typical sticking factors of approx. 0.1 for formic acid on copper at $300 \mathrm{~K} .{ }^{5}$ Based on the principle of microscopic reversibility, we therefore employed a method using only desorption trajectories that pass through the

a) Electronic mail: sjj24@cam.ac.uk transition state for formic acid dissociation on the copper surface. This method has previously been successfully applied to reveal vibrational mode specificity for the chemisorption of methane on metal surfaces. ${ }^{6-8}$

To determine the transition state necessary for initialising the MD simulations, the adsorption geometries of formic acid and formate on $\mathrm{Cu}\{531\}$ and $\mathrm{Cu}\{110\}$ first had to be determined. All DFT calculations have been performed with the CASTEP code (Version 18.1) ${ }^{9}$ using the PBE exchange-correlation functional, ${ }^{10}$ ultrasoft pseudopotentials ${ }^{11}$ and a cut-off energy of $500 \mathrm{eV}$ for the plane-wave basis. To account for long-range moleculesurface interactions, the Tkatchenko-Scheffler dispersion force correction scheme ${ }^{12}$ was utilised. More computational details can be found in the SI. Careful sampling of different initial geometries on the $\mathrm{Cu}\{531\}$ surface enabled identification of the most stable adsorption geometries for both formic acid and formate above the (110) microfacet, as shown in the left and right panels of Figure 1 (more details in Sec. 2 of the SI). To the best of our knowledge, the adsorption and dissociation of formic acid on $\mathrm{Cu}\{531\}$ have not previously been studied, experimentally or computationally.

When adsorbed on $\mathrm{Cu}\{110\}$, formate adopts a bidentate geometry, occupying the short bridge (sb) site of a close-packed row. ${ }^{13-15}$ Such an arrangement has been assumed here, and the hydrogen atom liberated upon adsorption was placed at an sb site of an adjacent closepacked row. As shown in the left panel of Figure 2, the most stable geometry of formic acid on $\mathrm{Cu}\{110\}$ exhibits its molecular plane parallel to the surface normal and perpendicular to the close-packed rows of $\mathrm{Cu}$, which is consistent with DFT calculations for formic acid on the structurally similar $\mathrm{Cu}\{211\}$ surface. ${ }^{16}$

Transition state searches connecting the most stable geometries of formic acid and formate, thus likely yielding the transition state that is lowest in energy and therefore most relevant for the desorption process, were initially surveyed with the LST-QST ${ }^{17}$ algorithm as implemented in CASTEP. These candidate transition state structures were then refined using hybrid eigenvectorfollowing ${ }^{18-20}$ in the OPTIM code in conjunction with CASTEP energies and gradients until the root-mean- 


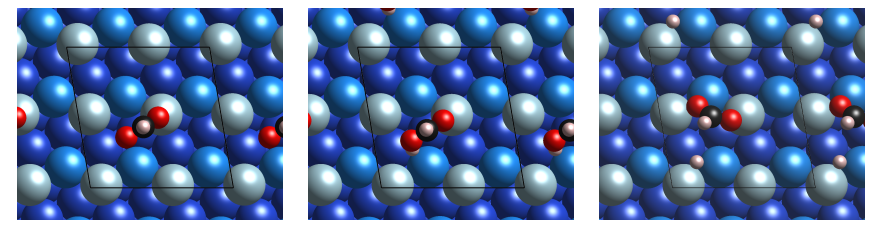

FIG. 1. Formic acid dissociation on R-Cu\{531\}, atoms shown in red (oxygen), black (carbon) and off-white (hydrogen) and shades of blue (copper; brightest closer to the surface). (Left) Formic acid, (middle) transition state, (right) formate and hydrogen after dissociation.
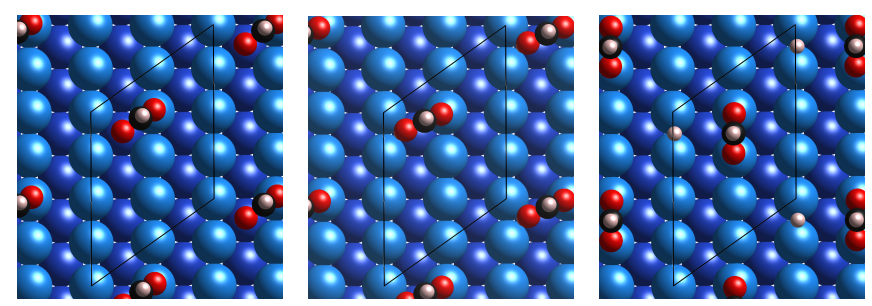

FIG. 2. Formic acid dissociation on $\mathrm{Cu}\{110\}$. (Left) Formic acid, (middle) transition state, (right) formate and hydrogen after dissociation.

square (RMS) gradient was smaller than $0.001 \mathrm{eV} \AA^{-1}$. Finite-displacement calculation of molecular vibrations revealed the expected normal modes and confirmed that only a single normal mode frequency was imaginary, representing the reaction coordinate (see SI for list of frequencies). The same amount of kinetic energy for each trajectory was distributed equally among these modes, i.e. $\mathrm{k}_{B} \mathrm{~T}$ in each normal mode apart from the reaction coordinate, which due to its single quadratic degree of freedom, was assigned only $\mathrm{k}_{B} \mathrm{~T} / 2(\mathrm{~T}=300 \mathrm{~K})$. This initialisation is based on the equipartition assumption for the transition state. The phase of each vibrational mode was chosen randomly, thus enabling the generation of different initial velocities for MD trajectories with the same amount of assigned kinetic energy. The key question that our work will address is whether asymmetry in the transition state survives the randomness of thermal desorption to generate a robust asymmetry in the eventual rotational ensemble. We will focus particularly upon the component of the angular momentum parallel to the surface normal, since the pseudovector nature of spin implies that a non-vanishing mean value of this component is the signature of desorption from a chiral surface (see SI).

MD simulations were performed using an NVE ensemble (constant number $\mathrm{N}$ of particles, volume $\mathrm{V}$, and total energy E) and a time step of 0.5 fs. A total of 32 trajectories were computed per surface, of duration 200 fs each, which allows for full desorption and an eventual separation between surface and molecule of around $4 \AA$. Along with the $\mathrm{k}_{B} \mathrm{~T}$ kinetic energy assignment to the modes, we noted that full desorption from the surface was only achieved if another $1.2 \mathrm{eV}$ of translational energy par- allel to the surface normal (z-axis of the supercell) was assigned to the molecular centre of mass. Here we focus on a subset of molecules from the high-energy tail of the Maxwell-Boltzmann energy distribution. These molecules with 'hyperthermal' kinetic energy ${ }^{6-8}$ associated with their surface-normal motion are precisely those that are most likely to fully desorb and be amenable to measurement in an experiment.
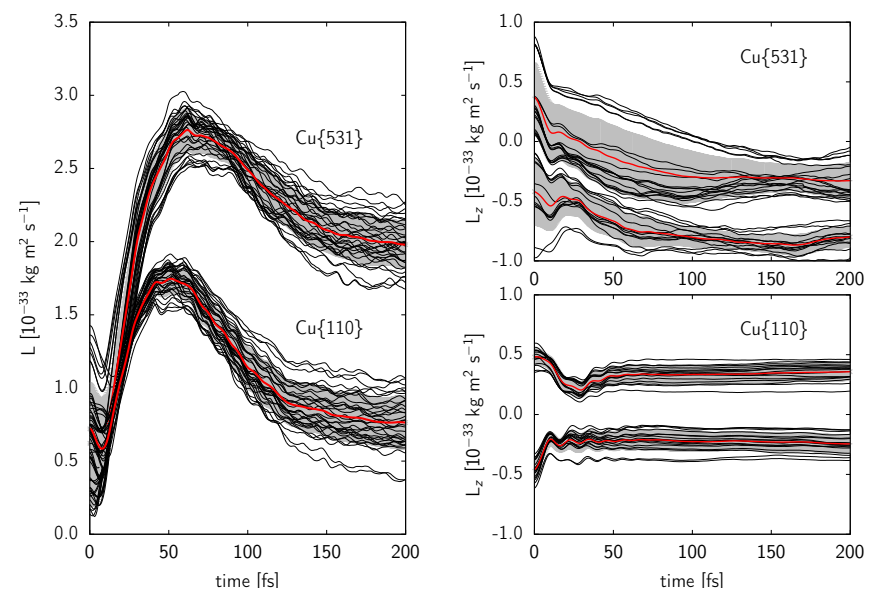

FIG. 3. Time evolution of the magnitude of the spin angular momentum, L (left) and z-component of $\mathbf{L}$ (right) for each trajectory on $\mathrm{Cu}\{531\}$ and $\mathrm{Cu}\{110\}$. Red lines indicate the mean values for each surface (left)/each population per surface (right), while an error range of one standard deviation is indicated in grey.

The angular momentum of the formic acid molecule, $\mathbf{L}$, is defined as

$$
\mathbf{L}=\sum_{i=1}^{N}\left(\mathbf{r}_{i} \times \mathbf{v}_{i}\right) m_{i}
$$

where $N$ is the number of atoms, $\mathbf{r}_{i}$ and $\mathbf{v}_{i}$ are the position and velocity vectors of atom $i$ (both relative to the centre of mass), and $m_{i}$ is its atomic mass. The left panel of Figure 3 shows the time evolution of the modulus of $\mathbf{L}$ for each trajectory on both surfaces, together with the mean for each set. It is evident that formic acid molecules desorbing from the two surfaces exhibit significantly different angular momenta after about 30 fs. On $\mathrm{Cu}\{531\}$ a much larger $\mathrm{L}$ value is observed, which persists over time, while the angular momentum on $\mathrm{Cu}\{110\}$ is smaller (even at its maximum) and afterwards decays gradually to a level similar to its initial value. The decline of the angular momentum from its maximum is larger, in both absolute and relative terms, for the achiral surface than for the chiral one. Note that the angular momentum is nonzero from the start because the vibrational modes are perturbed by the surface at the transition state (see SI for more computational details). The mean angular momentum is initially not much different between the two surfaces, but its slope changes rapidly. We note that the 

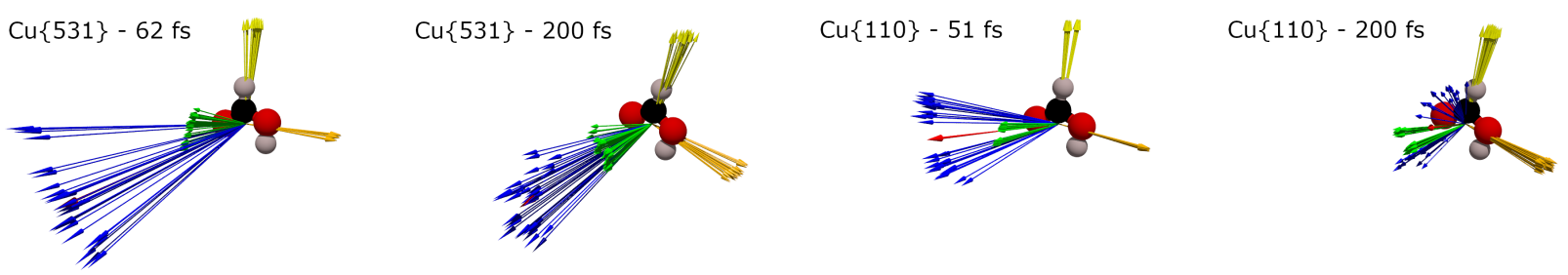

FIG. 4. Time evolution of the angular momentum vector (dark blue), its mean (red) and the principal axes of I (green - $\mathrm{I}_{C}$, yellow $-\mathrm{I}_{B}$, orange $-\mathrm{I}_{A}$ ) of formic acid desorbing from $\mathrm{Cu}\{531\}$ after $62 \mathrm{fs}$ and $200 \mathrm{fs}$, and from $\mathrm{Cu}\{110\}$ after $51 \mathrm{fs}$ and $200 \mathrm{fs}$.

rotational effect is comparatively small, associated with a maximum of $30 \mathrm{meV}$ kinetic energy. It is, however, the significant difference in both magnitude and time evolution of the molecular rotation descriptors between the desorption from chiral and achiral surfaces that is of interest here.

The two right panels of Figure 3 show that the zcomponent (parallel to the surface normal) of $\mathbf{L}$ is significantly different for the two surfaces. In each case the trajectories exhibit bimodal populations, with positive and negative initial $\mathrm{L}_{z}$. This bifurcation correlates very well with the random phase assignment of mode number 8 (see Table 3 in the SI) being either positive or negative. While the two populations on $\mathrm{Cu}\{110\}$ retain their opposing signs, $\mathrm{L}_{z}$ on $\mathrm{Cu}\{531\}$ declines below 0 for both populations and levels out at about -0.3 and -0.8 $\left(10^{-33} \mathrm{~kg} \mathrm{~m}^{2} \mathrm{~s}^{-1}\right)$, respectively. These values correspond to an overall tilt out of the surface plane of $10^{\circ}$ and $24^{\circ}$, and an average tilt of $15^{\circ}$ for $\mathrm{Cu}\{531\}$; the corresponding angles are $22^{\circ}$ and $27^{\circ}$ for the two populations on $\mathrm{Cu}\{110\}$, averaging to just $2^{\circ}$. Moreover, if one were to average including mirror images of the $\mathrm{Cu}\{110\}$ transition state studied here, the mean $\mathrm{L}_{z}$ component for the achiral system would vanish entirely. Lacking any degenerate mirror-image transition states, however, the same will not be true for desorption from the $\mathrm{Cu}\{531\}$ surface.

Another important descriptor of the rotational properties for a three-dimensional object is the moment of inertia and the corresponding principal axes. These axes are defined by the eigenvectors of the moment of inertia tensor, which is obtained from the positions of all atoms in the formic acid molecule, again with respect to the centre of mass. The diagonal elements of the moment of inertia tensor are defined as $I_{x x}=\sum_{i} m_{i}\left(r_{i}^{2}-x_{i}^{2}\right)$ and similarly for $I_{y y}$ and $I_{z z}$, using the Cartesian coordinates $x_{i}, y_{i}$ and $z_{i}$ of atom $i$. Off-diagonal elements are given by $I_{x y}=-\sum_{i} m_{i} x_{i} y_{i}$ etc.

The angular momentum vectors at two different time points of the trajectories for both surfaces, together with the respective principal axes of the moment of inertia and the molecule itself, are shown in Figure 4. In this depiction, all three principal axes of the moment of inertia are normalised to the same vector length. Taking the respective eigenvalues of these eigenvectors into account reveals that their magnitude ratio is actually $77: 67: 10$ for the green:yellow:orange axes (following convention, the labels $\mathrm{I}_{C}, \mathrm{I}_{B}$ and $\mathrm{I}_{A}$ will be used). Therefore, formic acid behaves as an asymmetric top, with its long axis (smallest moment of inertia) along $\mathrm{I}_{A}$ and its short axis (largest moment of inertia) along $\mathrm{I}_{C}$. To a reasonable approximation, however, one might alternatively consider it as a pseudo-prolate top, since $\mathrm{I}_{B}$ and $\mathrm{I}_{C}$ are quite similar in magnitude. Figure 4 clearly shows an alignment around $\mathrm{I}_{C}$, which is much more pronounced on $\mathrm{Cu}\{531\}$ than on $\mathrm{Cu}\{110\}$, and that only one rotational sense is present in the ensemble. Additionally, Figure 5 shows a projection of the angular momentum vectors onto a plane perpendicular to $\mathrm{I}_{C}$ at different times during the simulation.

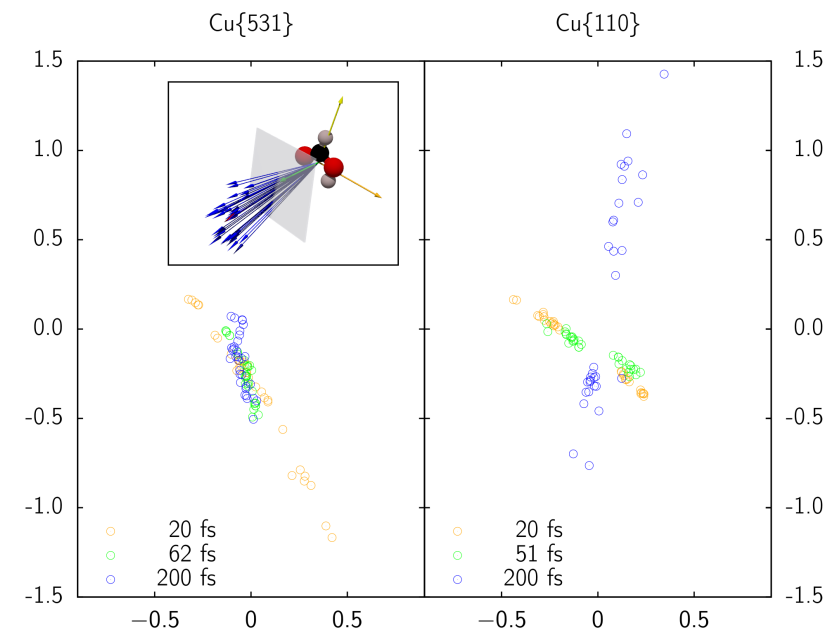

FIG. 5. Representation of the spread of angular momentum vectors for each desorption trajectory from $\mathrm{Cu}\{531\}$ and $\mathrm{Cu}\{110\}$ at $20 \mathrm{fs}$, the time of maximum angular momentum (62 fs and $51 \mathrm{fs}$, respectively) and at the end of the simulation (200 fs). The vectors were projected onto a plane (shown in grey in the inset as a schematic of the projection) normal to the $\mathrm{I}_{C}$ vector of the respective time and surface, located to be intersected by $\mathrm{I}_{C}$ at its normalised length. The $\mathrm{x}$ and $\mathrm{y}$ directions are chosen to be parallel to $\mathrm{I}_{A}$ and $\mathrm{I}_{B}$ for each data set.

Initially, the molecule experiences a kick from the formation of the $\mathrm{O}-\mathrm{H}$ bond, which occurs in roughly the first 10 fs of each trajectory. The induced angular momentum has a significant component along $\mathrm{I}_{A}$ with no preference for a particular rotational sense around that axis and only a small $\mathrm{I}_{C}$ component. Over roughly the next 50 (40) fs the angular momentum vector associated with 
desorption from $\mathrm{Cu}\{531\}(\mathrm{Cu}\{110\})$ increases in magnitude and aligns increasingly with $\mathrm{I}_{C}$, see Figure 4 . Later in the calculated trajectories, the magnitude of the angular momentum decreases, while the $\mathrm{I}_{A}$ component of the vector continues to fall. The decrease in magnitude must be attributed to a surface-induced torque. Assuming that the main surface-adsorbate interaction is that between the carbonyl oxygen and the topmost copper atom to which it is initially bonded, we propose that the deceleration of the molecular rotation stems from the torque exerted while that bond is progressively broken. As the distance to the surface increases, the interaction strength between $\mathrm{O}$ and $\mathrm{Cu}$ gradually declines and eventually disappears, as does its decelerating effect on the molecular rotation. After $200 \mathrm{fs}$, the surface-adsorbate interaction is negligible and the angular momentum should be unchanged thereafter (but see SI for a discussion of interacting periodic images), especially because $\mathrm{I}_{C}$ is the principal axis with the largest moment of inertia and rotations around that axis are stable against small variations. ${ }^{21}$ Isolated, freely rotating molecules will furthermore tend to align their axis of rotation with their respective $\mathrm{I}_{C}$ axis over time due to energy dissipation and angular momentum conservation. This could very well have caused the molecules in this study to align with $\mathrm{I}_{C}$ whilst still interacting with the surface, but this will have to be subject to further studies.

Our main result is that all angular momentum vectors aligned along $\mathrm{I}_{C}$ exhibit the same sense of rotation upon desorption from $\mathrm{Cu}\{531\}$. This result indicates a clear overall preference for this sense of rotation, as no mirror image of the transition state can exist. Applying the principle of microscopic reversibility, this result translates into a higher probability for molecules with this rotational sense to enter the most energetically favourable reaction pathway for dissociative adsorption on a surface of corresponding chirality. As net chirality has been shown to persist even when idealised surfaces are modified by surface roughening, ${ }^{22,23}$ this effect might also be observable on defective and thermally roughened chiral surfaces. On $\mathrm{Cu}\{110\}$, however, mirror-image transition states are equally likely, and so molecules with the opposite sense of rotation should be connected with symmetry related transition states. Additionally, the larger $\mathrm{I}_{B}$ component in the angular momentum vectors on $\mathrm{Cu}\{110\}$ (see Figure 5, right panel) and the rapid decay of the angular momentum, shows that there is much less of a significant, directed effect on the achiral surface even when only a single transition state is considered.

In conclusion, we have demonstrated a relationship between structural surface chirality and chiral motion of desorbing molecules, comparing the desorption of formic acid from the chiral $\mathrm{Cu}\{531\}$ and achiral $\mathrm{Cu}\{110\}$ surfaces. Following the identification of the most energetically favourable reaction pathway for dissociative adsorption on both surfaces, we performed MD simulations of formic acid desorption and analysed the time evolution of rotational properties for desorbing molecules. On the chiral surface, a much larger and more directed effect on the molecular rotation was observed, with only one rotational sense, a significant and non-vanishing surfacenormal component, and a much narrower spread of the angular momentum vector compared to the achiral surface. According to the principle of microscopic reversibility, these observations also hold true in time-reversal, i.e. upon adsorption. This study therefore provides new insight into the influence of rotation on adsorption probabilities and in particular the likely influence of surface chirality on such processes. We find that achiral reactants with one sense of molecular rotation, which have been shown to be experimentally accessible, ${ }^{24}$ should be much more likely to undergo adsorption and reaction processes on a chiral substrate than those with the opposite rotational sense. This effect has the potential to open up entirely new applications for chiral surfaces and inspire the design of new experiments.

\section{ACKNOWLEDGMENTS}

We express our thanks to Dr Marco Sacchi for helpful discussions. SCM gratefully acknowledges financial support from UKRI/EPSRC under grant number 2112388. DJW thanks the EPSRC for funded support.

${ }^{1}$ M. Kuroha, S. Nambu, S. Hattori, Y. Kitagawa, K. Niimura, Y. Mizuno, F. Hamba, and K. Ishii, "Chiral Supramolecular Nanoarchitectures from Macroscopic Mechanical Rotations: Effects on Enantioselective Aggregation Behavior of Phthalocyanines," Angew. Chem. Int. Ed. 58, 18454-18459 (2019).

${ }^{2}$ L. Vattuone, L. Savio, F. Pirani, D. Cappelletti, M. Okada, and M. Rocca, "Interaction of rotationally aligned and of oriented molecules in gas phase and at surfaces," Prog. Surf. Sci. 85, 92160 (2010).

${ }^{3}$ A. Gerbi, L. Savio, L. Vattuone, F. Pirani, D. Cappelletti, and M. Rocca, "Role of rotational alignment in dissociative chemisorption and oxidation: $\mathrm{O}_{2}$ on bare and CO-precovered Pd(100)," Angew. Chem. Int. Ed. 45, 6655-6658 (2006).

${ }^{4}$ S. J. Jenkins, Chirality at Solid Surfaces (John Wiley \& Sons, Ltd, Chichester, UK, 2018).

${ }^{5}$ M. Bowker, S. Haq, R. Holroyd, P. M. Parlett, S. Poulston, and N. Richardson, "Spectroscopic and kinetic studies of formic acid adsorption on $\mathrm{Cu}(110)$," J. Chem. Soc. Faraday Trans. 92, 4683 (1996), arXiv:1011.1669v3.

${ }^{6}$ M. Sacchi, D. J. Wales, and S. J. Jenkins, "Mode-Specific Chemisorption of $\mathrm{CH}_{4}$ on $\mathrm{Pt}\{110\}-(1 \times 2)$ Explored by FirstPrinciples Molecular Dynamics," J. Phys. Chem. C 115, 2183221842 (2011).

${ }^{7}$ M. Sacchi, D. J. Wales, and S. J. Jenkins, "Mode-specificity and transition state-specific energy redistribution in the chemisorption of $\mathrm{CH}_{4}$ on $\mathrm{Ni}\{100\}$," Phys. Chem. Chem. Phys. 14, 15879 (2012).

${ }^{8}$ M. Sacchi, D. Wales, and S. Jenkins, "Bond-selective energy redistribution in the chemisorption of $\mathrm{CH}_{3} \mathrm{D}$ and $\mathrm{CD}_{3} \mathrm{H}$ on Pt $\{110\}-(1 \times 2)$ : A first-principles molecular dynamics study," Comput. Theor. Chem. 990, 144-151 (2012).

${ }^{9}$ S. J. Clark, M. D. Segall, C. J. Pickard, P. J. Hasnip, M. I. J. Probert, K. Refson, and M. C. Payne, "First principles methods using CASTEP," Zeitschrift für Krist. - Cryst. Mater. 220, 567570 (2005).

${ }^{10}$ J. P. Perdew, K. Burke, and M. Ernzerhof, "Generalized Gradient Approximation Made Simple," Phys. Rev. Lett. 77, 3865-3868 (1996). 
${ }^{11}$ D. Vanderbilt, "Soft self-consistent pseudopotentials in a generalized eigenvalue formalism," Phys. Rev. B 41, 7892-7895 (1990).

${ }^{12}$ A. Tkatchenko and M. Scheffler, "Accurate Molecular Van Der Waals Interactions from Ground-State Electron Density and Free-Atom Reference Data," Phys. Rev. Lett. 102, 073005 (2009).

${ }^{13}$ A. Chutia, I. P. Silverwood, M. R. Farrow, D. O. Scanlon, P. P. Wells, M. Bowker, S. F. Parker, and C. R. A. Catlow, "Adsorption of formate species on $\mathrm{Cu}(\mathrm{h}, \mathrm{k}, \mathrm{l})$ low index surfaces," Surf. Sci. 653, 45-54 (2016).

${ }^{14}$ D. Kreikemeyer-Lorenzo, W. Unterberger, D. A. Duncan, M. K. Bradley, T. J. Lerotholi, J. Robinson, and D. P. Woodruff, "Facedependent bond lengths in molecular chemisorption: The formate species on $\mathrm{Cu}(111)$ and $\mathrm{Cu}(110)$," Phys. Rev. Lett. 107, 046102 (2011).

${ }^{15}$ H. Nakano, I. Nakamura, T. Fujitani, and J. Nakamura, "Structure-Dependent Kinetics for Synthesis and Decomposition of Formate Species over $\mathrm{Cu}(111)$ and $\mathrm{Cu}(110)$ Model Catalysts," J. Phys. Chem. B 105, 1355-1365 (2001).

${ }^{16} \mathrm{~S}$. Li, J. Scaranto, and M. Mavrikakis, "On the Structure Sensitivity of Formic Acid Decomposition on Cu Catalysts," Top. Catal. 59, 1580-1588 (2016).

${ }^{17}$ N. Govind, M. Petersen, G. Fitzgerald, D. King-Smith, and J. Andzelm, "A generalized synchronous transit method for transition state location," Comput. Mater. Sci. 28, 250-258 (2003).

${ }^{18}$ L. J. Munro and D. J. Wales, "Defect migration in crystalline silicon," Phys. Rev. B 59, 3969-3980 (1999).
${ }^{19}$ G. Henkelman and H. Jónsson, "A dimer method for finding saddle points on high dimensional potential surfaces using only first derivatives," J. Chem. Phys. 111, 7010-7022 (1999).

${ }^{20}$ Y. Kumeda, D. J. Wales, and L. J. Munro, "Transition states and rearrangement mechanisms from hybrid eigenvector-following and density functional theory." Chem. Phys. Lett. 341, 185-194 (2002).

${ }^{21}$ L. Landau and E. Lifshitz, "Motion of a Rigid Body," in Mechanics (Elsevier, 1976) pp. 96-130.

22 A. E. Baber, A. J. Gellman, D. S. Sholl, and E. C. H. Sykes, "The Real Structure of Naturally Chiral Cu\{643\}," J. Phys. Chem. C 112, 11086-11089 (2008).

${ }^{23}$ M. L. Clegg, S. M. Driver, M. Blanco-Rey, and D. A. King, "Atomic roughness of an intrinsically chiral surface orientation of an FCC metal: $\mathrm{Cu}\{531\}$," J. Phys. Chem. C 114, 4114-4117 (2010).

${ }^{24}$ S. Fleischer, Y. Khodorkovsky, Y. Prlor, and I. S. Averbukh, "Controlling the sense of molecular rotation," New J. Phys. 11 (2009).

${ }^{25}$ M. E. Straumanis and L. S. Yu, "Lattice parameters, densities, expansion coefficients and perfection of structure of $\mathrm{Cu}$ and of Cu-In $\alpha$ phase," Acta Crystallogr. Sect. A 25, 676-682 (1969).

${ }^{26} \mathrm{H}$. J. Monkhorst and J. D. Pack, "Special points for Brillouinzone integrations," Phys. Rev. B 13, 5188-5192 (1976).

${ }^{27}$ G. Kresse and J. Furthmüller, "Efficiency of ab-initio total energy calculations for metals and semiconductors using a plane-wave basis set," Comput. Mater. Sci. 6, 15-50 (1996). 